Topics

\title{
Development of a Laser-Electrostatic Hybrid Acceleration Propulsion System
}

\author{
By Tatsuro SAKAI, Akihiro OSAMURA and Hideyuki HorISAWA \\ Tokai University, Hiratsuka, Japan
}

(Received June 24th, 2013)

\begin{abstract}
The speed and number of ions in a laser-electrostatic hybrid thruster were experimentally estimated using a Faraday cup for various electrode configurations to determine the optimum electrode geometry. The results showed that, for a 10-mm-diameter electrode placed in front of a laser ablation target, the speed of ions increased with target voltages. The ion speeds, measured $80 \mathrm{~mm}$ away from the target, were $16 \mathrm{~km} / \mathrm{s}$ and $32 \mathrm{~km} / \mathrm{s}$ for target voltages of $0 \mathrm{~V}$ and $500 \mathrm{~V}$, respectively, for a target-electrode gap of $20 \mathrm{~mm}$.
\end{abstract}

Key Words: Microchip Laser, Laser Ablation, Laser-Electric Hybrid Propulsion, Electrostatic Acceleration

\section{Nomenclature}

$$
\begin{array}{lll}
J & : & \text { Discharge current } \\
B & : & \text { Induced magnetic field } \\
Z & : & \text { Target-acceleration electrode gap } \\
V_{t g} & : & \text { Target voltage }
\end{array}
$$

\section{Introduction}

Small-sized onboard laser plasma thrusters are under significant development with rapid evolutions of compact, high-power laser systems ${ }^{1-3}$. One advantage of such newly developed laser thrusters is the use of solid-state materials as propellants. Because these laser propulsion systems use solid propellants, they do not require tanks, valves, or piping systems, which are necessary for thrusters with liquid or gaseous propellants. Therefore, such laser thruster systems can have simple and compact designs. In addition, the thrust can be significantly controlled by varying the input laser power. We had previously conducted a preliminary study on a laser-electric hybrid propulsion system ${ }^{4-7)}$ to further improve the thrust performances and simplify conventional laser propulsion systems.

A schematic of our laser-electric hybrid acceleration system is shown in Fig. 1. The basic principle of laser ablation thrusters is that a laser ablation plasma, induced through laser irradiation on a solid target, is further accelerated using an accelerator grid. Because the laser ablation plasma of initial velocity of $\sim 15 \mathrm{~km} / \mathrm{s}^{6,7)}$, is further accelerated by through an electrostatic or electromagnetic method, the thrust and specific impulses can be significantly increased.

\subsection{Laser-electric hybrid acceleration thrusters}

In laser-electric hybrid acceleration thrusters, the acceleration regimes of the laser ablation plasma can be classified into three types, according to the electrode

configuration, plasma density, and electrical input power (voltage and current), i) electrostatic acceleration, ii) electromagnetic acceleration, and iii) electrothermal

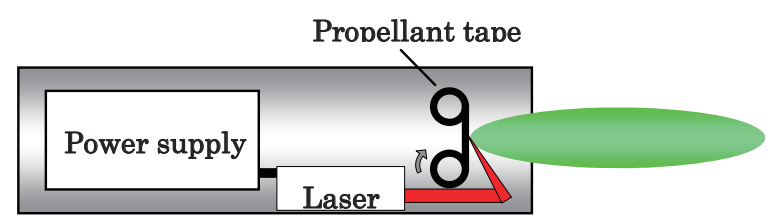

(a)

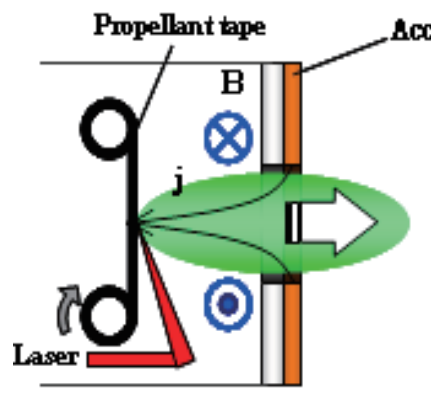

(b)

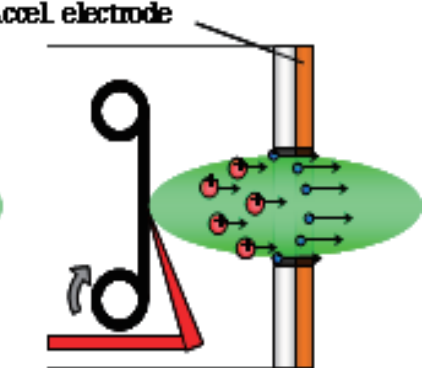

(c)
Fig 1. Schematics of laser ablation thrusters; (a) pure laser thruster, (b) laser-electromagnetic hybrid thruster, and (c) laser-electrostatic hybrid thruster.

acceleration. Especially for the laser ablation plasma, depending on laser conditions such as pulse energy, fluence, etc., plasma density distribution can be widely controlled. Moreover, this can also be increased using additional electric discharges.

Therefore, by optimizing the electrode configuration to facilitate additional electric acceleration and by controlling the power source (voltage and current), a propulsion system that adapts to all the aforementioned acceleration regimes can be constructed using one thruster configuration. Such a system enables a robust conversion between high specific impulse operation and high thrust density operation according to mission requirements. The two types of acceleration schemes 
that are currently being investigated are described in the following subsections.

\subsection{Laser-electromagnetic hybrid acceleration mode}

One laser-electric hybrid acceleration mode considered in our recent studies is the laser-electromagnetic acceleration. In order to improve the thrust performances of electromagnetic acceleration thrusters such as pulsed-plasma thrusters (PPTs), the effects of using a laser beam are being investigated.

Although the PPTs using solid propellants usually PTFE (Teflon ${ }^{\circledR}$ ), have a low efficiency, they have attracted increasing attention owing to their system simplicity and advantages of miniaturization and mass reduction for use as altitude or orbit control thrusters in small-sized spacecrafts ${ }^{8-10)}$. In such PPTs, it is difficult to complete the process in which phase changes and electromagnetic acceleration simultaneously occur during a discharge pulse owing to a delay in phase changes of the solid propellant after the pulse discharge initiation. Various masses including low-speed macroparticles can have significantly different velocities. Owing to the residual vapor or plasma in the discharge chamber resulting from the delayed evaporation of the propellant surface, which cannot contribute to the impulse bit, improving this loss of propellant mass and, consequently, thrust efficiency has been difficult ${ }^{7-9)}$. In order to minimize this delayed ablation and to improve the thrust efficiency, the effects of using of laser-pulse irradiation, or assistance, were investigated. Such laser-pulse irradiation can induce a conductive plasma from a solid-propellant surface in a short duration, i.e., by using a short-duration conductive region of the plasma between the electrodes, short-pulse switching or discharge can be achieved ${ }^{4-6}$. Because the use of a shorter pulsed laser results in a shorter pulsed-plasma duration in this case, a higher peak current and significant improvement in the thrust performances can be expected. In addition, depending on the laser power the laser-induced plasma resulting from a solid-propellant usually has a directed initial velocity, which can also improve the thrust performance compared to conventional PPTs. A schematic of a coaxial laser-electromagnetic acceleration thruster is illustrated in Fig. $1(\mathrm{~b})^{6}$.

\subsection{Laser-electrostatic acceleration mode}

We conducted a preliminary investigation on a laser-electrostatic hybrid acceleration thruster, in which the laser ablation plasma was accelerated through an electrostatic field $^{7)}$. The mechanism of this mode is as follows: firstly, a focused laser pulse is irradiated on to a solid target (the propellant). Then, a laser-induced plasma, or laser ablation is produced at the irradiated spot of the propellant surface. In the laser ablation process, the electrons are first emitted from the surface, and ions are then accelerated through ambipolar diffusion ${ }^{6,7)}$. These ions are further accelerated using an additional acceleration electrode. Because the laser-induced plasma having a directed initial velocity is further accelerated through an electrostatic field, a high specific-impulse can be expected. In this mode, a neutralizer is required because no electrons are ejected. However, in this study, no neutralizer was utilized for simplicity of the investigation of the whole phenomena, since the phenomena are strongly dominated by the pulsed ambipolar diffusion of electrons and ions.

The objective of this study was to investigate the optimum electrode configurations and to observe the acceleration characteristics of the laser-electrostatic hybrid acceleration mode.

\section{Experimental Apparatus}

A schematic illustration of the laser-electrostatic hybrid thruster is shown in Fig. 2. The hybrid thruster consists of a $\mathrm{Cu}$ target (propellant), a pair of electrodes, and a laser oscillator. The $\mathrm{Cu}$ target was mounted on an $\mathrm{X}-\mathrm{Y}$ stage to refresh the irradiated surfaces. For the laser oscillator, an LD-pumped Nd:YAG microchip laser (JDS UNIPHASE, PowerChip Nanolaser; wavelength: $1064 \mathrm{~nm}$; pulse energy: 50 $\mu \mathrm{J} /$ pulse; pulse width: $250 \mathrm{ps}$; repetition rate: $1 \mathrm{kHz}$ ) was used. Laser ablation plasmas or ions were accelerated by the acceleration electrodes consisting of $1 \mathrm{~mm}$-thick $\mathrm{Cu}$ plates with a hole of $10 \mathrm{~mm}$ in diameter.

Based on our previous measurements ${ }^{7)}$ of electron temperatures and densities of a laser ablation plasma for the same configurations, a range of distances ( $Z$ : target acceleration electrode gap) and a distance of $1 \mathrm{~mm}$ between the electrodes were chosen for the effective electrostatic acceleration of ions. The first electrode (inner electrode) was electrically floated to enable its potential to be consistent the plasma potential. The second electrode (outer electrode) was biased from 0 to $-500 \mathrm{~V}$ relative to the target potential.

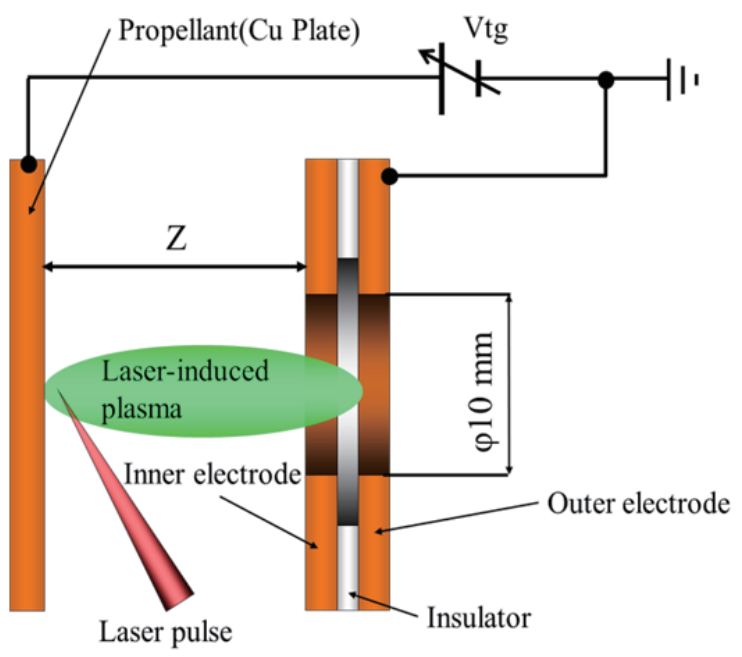

Fig. 2. Laser-electrostatic hybrid thruster.

The speed and the number of the ions exhausted from the thruster were measured using a Faraday cup. The experimental apparatus is shown in Fig. 3. The Faraday cup was placed 50 and $80 \mathrm{~mm}$ away from the surface of the ablation target. The ion current was amplified using an amplifier (DC to $10 \mathrm{MHz}$ ) and was monitored using an oscilloscope (Tektronix, TDS3034B; sampling rate: $2.5 \mathrm{GS} / \mathrm{s}$; frequency bandwidth: $300 \mathrm{MHz}$ ). The ion velocity distributions were calculated by dividing the distance between the target and the Faraday cup 


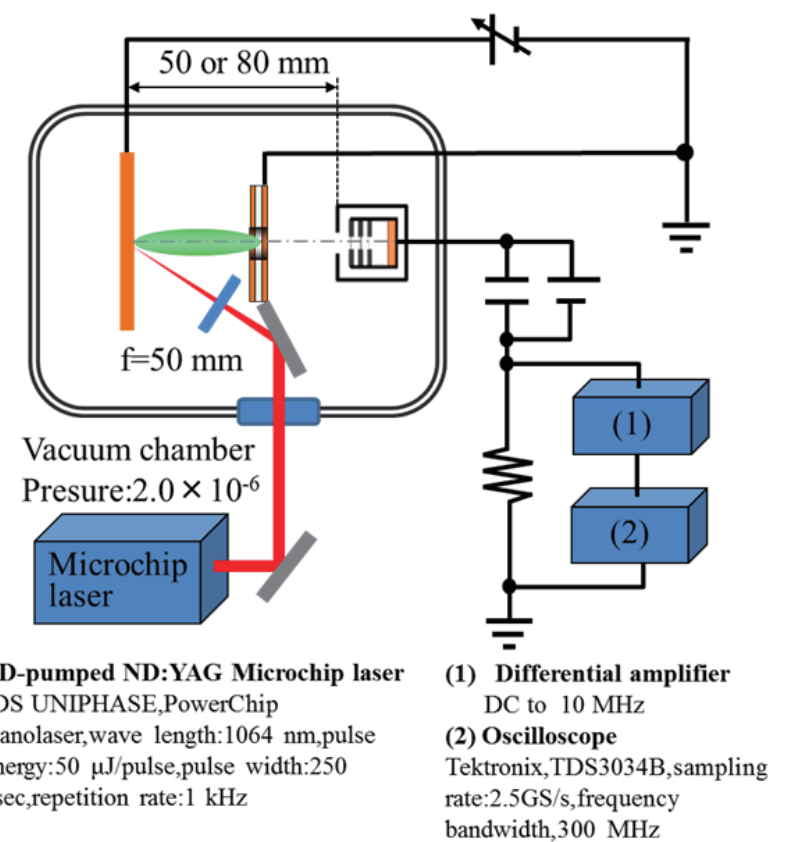

Fig. 3. Experimental setup.

by the time-of flight of the ions. The mean velocities of the ions were calculated by integrating the ion velocity distributions. In addition, the average ion speeds were also estimated from the delays of the peak ion currents of the Faraday cups located at different positions. These measurements indicated that the mean velocities of the ions estimated at the two positions were consistent with the average ion speeds.

\section{Results and Discussion}

The ion velocities measured using Faraday cup are plotted in Figs. 4 and 5, in which the Faraday cup distances were 50 and $80 \mathrm{~mm}$, respectively. For both the cases, the target

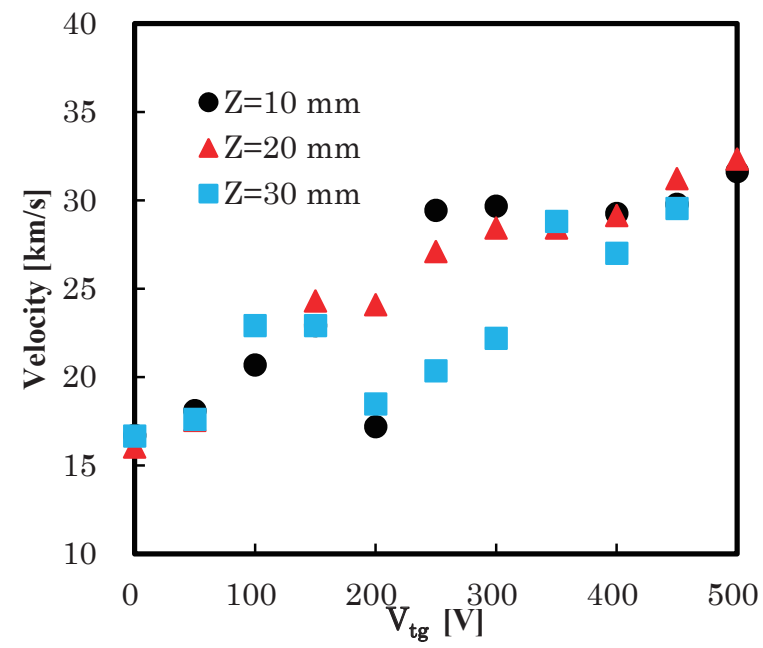

Fig. 4. Variations in the ion velocity with respect to the target voltage with the Faraday cup placed $50 \mathrm{~mm}$ away from the target.

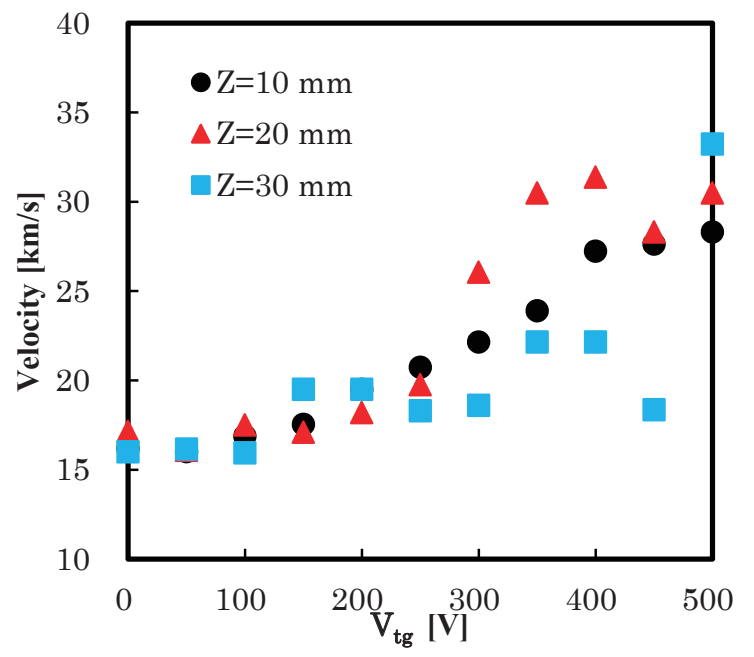

Fig. 5. Variations in the ion velocity with respect to the target voltage with the Faraday cup placed $80 \mathrm{~mm}$ away from the target.

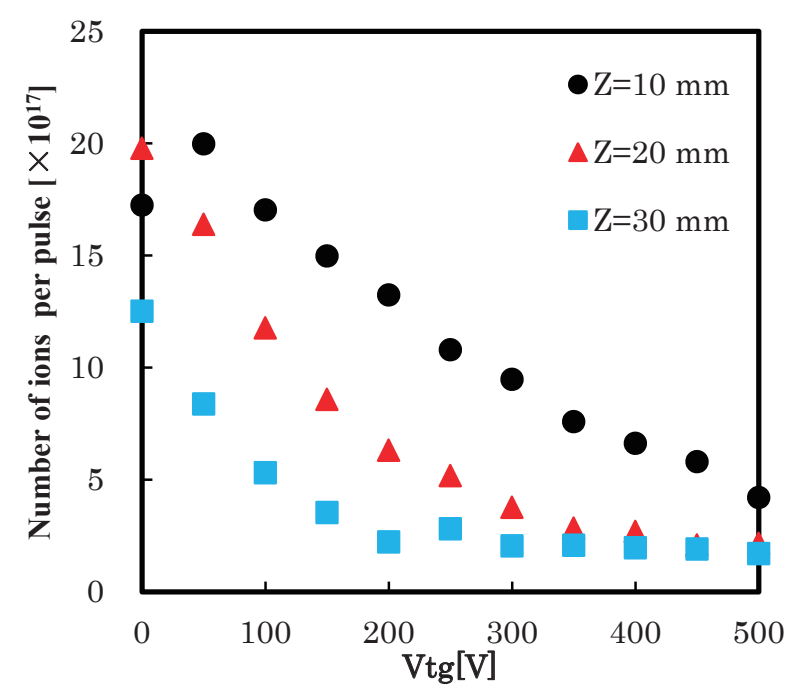

Fig. 6. Variations in the ion number with respect to the target voltage with the Faraday cup placed $50 \mathrm{~mm}$ away from the target.

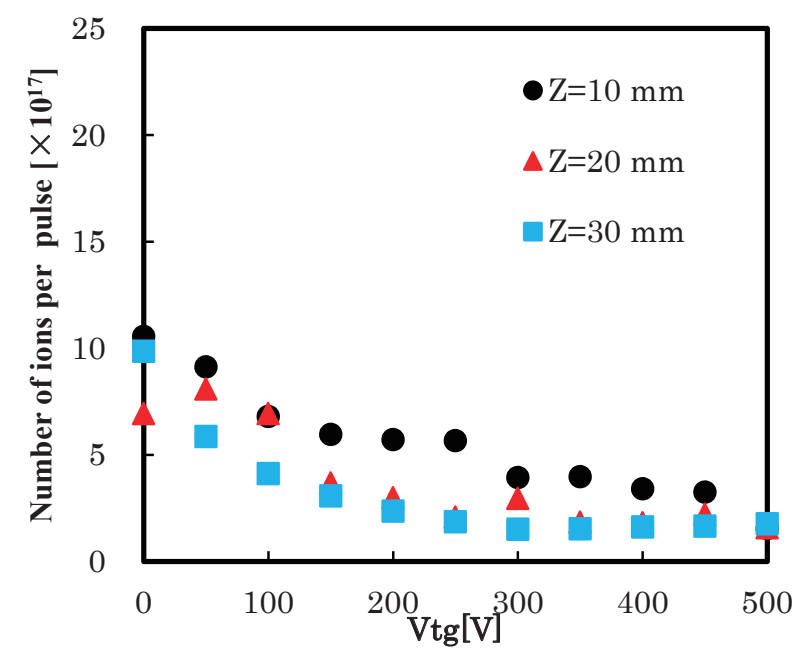

Fig. 7. Variations in the ion velocity with respect to the target voltage with the Faraday cup placed $80 \mathrm{~mm}$ away from the target. 
voltages i.e., the voltages of the target relative to those of the outer electrode, $V_{\mathrm{tg}}$, were increased from 0 to $+500 \mathrm{~V}$ to produce a negative electrostatic field to accelerate the positive ions for $Z=10$ to $30 \mathrm{~mm}$. The differences in the ion velocity profiles between Figs. 4 and 5 are probably due to the misalignment between the Faraday cup axis and the ion beam axis.

In Fig. 4, the ion velocities measured $50 \mathrm{~mm}$ away from the target increase with target voltages for all values of $Z$. The ion velocities were $17 \mathrm{~km} / \mathrm{s}$ and $30 \mathrm{~km} / \mathrm{s}$ for $V_{\mathrm{tg}}=0$ and $500 \mathrm{~V}$, respectively at $Z=20 \mathrm{~mm}$.

In Fig. 5, similar tendencies are observed for the ion velocities measured $80 \mathrm{~mm}$ away from the target. From the figure, the ion velocities are $16 \mathrm{~km} / \mathrm{s}$ and $32 \mathrm{~km} / \mathrm{s}$, respectively for $V_{\mathrm{tg}}=0$ and $500 \mathrm{~V}$ at $Z=20 \mathrm{~mm}$. Although some data scatters can be seen, the reproducibility of these data was within $10 \%$. Considering a theoretical ion velocity of $35 \mathrm{~km} / \mathrm{s}$, assuming singly ionized cupper ions accelerated in a potential of $-500 \mathrm{~V}$ (assuming the initial velocity of $0 \mathrm{~km} / \mathrm{s}$ ), the measured value of $32 \mathrm{~km} / \mathrm{s}$ is consistent with the theoretical value within the error.

The variations in the number of ion per pulse with respect to the target voltages measured using the Faraday cup are plotted in Figs. 6 and 7, in which the target-Faraday cup distances are 50 and $80 \mathrm{~mm}$, respectively. In both the figures, the number of ions decreased with increasing target voltages for all $Z$ values. This is probably caused by the enhanced diffusion of the ions owing to the larger potential of the acceleration electrode. In addition, the number of ions decreased with increasing target-Faraday cup distances. Further, when the electrode gap $Z$ increased, the number of ions decreased. Because the plasma diffused radially for larger $Z$ values, the number of ions traversing through the hole in the acceleration electrode decreased.

\section{Conclusion}

We experimentally estimated the speed and number of ions in a laser-electrostatic hybrid thruster using a Faraday cup for various electrode configurations and determined the optimum electrode geometry.

We observed that, for 10-mm-diameter electrodes placed 20 $\mathrm{mm}$ away from a laser ablation target, the speed of ions increased with voltages. The typical ion speeds, measured 80 $\mathrm{mm}$ away from the target, were $16 \mathrm{~km} / \mathrm{s}$ and $32 \mathrm{~km} / \mathrm{s}$ for target voltages of $0 \mathrm{~V}$ and $500 \mathrm{~V}$, respectively, with a target-electrode gap of $20 \mathrm{~mm}$.

In addition, the number of ions per pulse decreased with increasing target voltages for all target-electrode distances. Further, number of ions decreased with increasing target-Faraday cup distances. Moreover, the number of ions decreased with increasing target-electrode distances.

\section{References}

1) Phipps, C. Luke, J.: Diode Laser-driven Microthrusters: A New Departure for Micropropulsion, AIAA J., 40 (2000), pp.310-318.

2) Pakhomov, V. A. and Gregory, D. A.: Ablative Laser Propulsion: An Old Concept Revisited, AIAA J., 38 (2000), pp.725-727.

3) Phipps, C., Luke, J. R., Lippert, T., Hauer, M. and Wokaun, A.: Micropropulsion Using a Laser Ablation Jet, Journal of Propulsion and Power, 20 (2004), pp.1000-1011.

4) Horisawa, H., Igari, A., Kawakami, M. and Kimura, I.: Discharge Characteristics of Laser-Electric Hybrid Thrusters, AIAA Paper, 2004, pp.2004-3937.

5) Horisawa, H., Kawakami, M., Kimura, I.: Laser-Assisted Pulsed Plasma Thruster for Space Propulsion Applications, Applied Physics A., 81 (2005), pp.303-310.

6) Horisawa, H., Sasaki, K., Igari, A. and Kimura, I.: Laser-Electric Hybrid Acceleration System for Space Propulsion Applications, The Review of Laser Engineering, 34 (2006), pp.435- 441.

7) Ono, T., Uchida, Y., Horisawa, H. and Funaki, I.: Measurement of Ion Acceleration Characteristics of a Laser-Electrostatic Hybrid Microthruster for Space Propulsion Applications, Vacuum, 83 (2009), pp.213-216.

8) Jahn, R. G.: Physics of Electric Propulsion, McGraw-Hill, Ohio, 1968, pp.198-316.

9) Martinez-Sanchez, M.: Spacecraft Electric Propulsion - An Overview, Journal of Propulsion and Power, 14 (1998), pp. 688-699.

10) Burton, R. L. and Turchi, P.: Pulsed Plasma Thruster, Journal of Propulsion and Power, 14 (1998), pp.716-735. 